\title{
Identification of Biotinylated Molecules Using a Baculovirus-Expressed Luciferase-Streptavidin Fusion Protein
}

BioTechniques 20:452-459 (March 1996)

M. Karp, C. Lindqvist ${ }^{1}, \mathbf{R}$. Nissinen, S. Wahlbeck ${ }^{1}, \mathrm{~K}$. Åkerman' ${ }^{1}$ and C. Oker-Blom ${ }^{2}$ University of Turku, Turku; 1 Åbo Akademi University, Turku; and ${ }^{2}$ VTT Biotechnology and Food Research, Espoo, Finland

\section{ABSTRACT}

A genetic fusion between streptavidin of Streptomyces avidinii and luciferase of Pyrophorus plagiophthalamus was constructed. The fusion protein was produced in the Sf9 insect cell line using the baculovirus expression vector system (BEVS). Sodium dodecyl sulfate polyacrylamide gel electrophoresis of the proteins from cells infected with the recombinant virus, VL1393LucGR-StreptAv, revealed that the fusion protein migrated with an apparent molecular weight of $75 \mathrm{kDa}$. Light emission measurements showed that the infected cells produced about $255 \mathrm{mg}$ of the chimeric protein per liter of cell culture $\left(127.5 \mu \mathrm{g} / 1 \times 10^{6}\right.$ cells). Precipitation of the LucGR-StreptAv fusion protein with biotinylated acrylic beads as well as immunoblot analyses using biotinylated immunoglobulins indicated that both fusion moieties of the chimeric protein product were functional with respect to their physical and enzymatic activities.

\section{INTRODUCTION}

Streptavidin produced by the soil bacterium, Streptomyces avidinii, is a tetrameric protein where each subunit is capable of binding one molecule of biotin. The gene encoding streptavidin has been cloned and sequenced (2), and some chimeric proteins, such as streptavidin-protein A of Staphylococcus aureus, have been constructed and expressed in Escherichia coli (19). In addition, avidin that is both structurally and functionally homologous to streptavidin has been recently produced in E. $\operatorname{coli}(1)$.

Genes coding for various light-producing enzymes, luciferases, of bacterial as well as of insect origin have been previously expressed in both prokaryotes and eukaryotic organisms (4-6,8). We have used these genes as genetic reporters and for FACS-applications (9) as well as for different diagnostic applications such as production of reagents useful for enzyme-linked immunosorbent assays (ELISA) (15). The latter was conducted by constructing a genetic fusion of the genes encoding luciferase (LucGR) of the insect Pyrophorus plagiophthalamus, a Jamaican click beetle, and protein A of $S$. aureus.

The baculovirus expression vector system (BEVS) utilizes the strong polyhedrin gene promoter of the Autographa californica nuclear polyhedrosis virus (AcNPV). The genes of interest are inserted under the transcriptional regulation of the corresponding gene promoter, and the recombinant proteins are produced in lepidopteran insect cell cultures or larvae during viral infection $(11,12,20)$. In most cases, the protein products have turned out to be processed, similarly if not identically when compared to their authentic counterparts, thereby retaining their biological activity. Here, the abundant production of a LucGR-streptavidin fusion protein in Spodoptera frugiperda insect cells using the baculovirus system is reported.

\section{MATERIALS AND METHODS}

\section{Cells and Virus}

The lepidopteran insect $S$. frugiper$d a$ cell line (Sf9) was maintained as previously described $(16,20)$. The cells were grown at $27^{\circ} \mathrm{C}$ in TNM-FH culture medium (Sigma Chemical, St. Louis, MO, USA) supplemented with $10 \%$ fetal bovine serum, $100 \mathrm{U} / \mathrm{mL}$ penicillin, $40 \mu \mathrm{g} / \mathrm{mL}$ streptomycin, and $2.5 \mu \mathrm{g} / \mathrm{mL}$ Fungizone ${ }^{\circledR}$ (Life Technologies, Gaithersburg, MD, USA) either in suspension or as monolayer cultures.

A recombinant baculovirus containing the streptavidin-luciferase fusion construct under the transcriptional regulation of the polyhedrin gene promoter (PHP) was produced by co-transfection of the plasmid pVL1393-LucGR-StreptAv (Figure 1) and wild-type AcMNPV (strain E2) DNA into Sf9 cells. Isolation, purification and propagation of the recombinant virus were performed according to standard procedures $(16,20)$ combined with light emission measurements as previously described for VL1392-LucGR and Ac701-ProtA-LucGR $(6,9,14)$.

\section{Construction of Plasmids}

The $P$. plagiophthalamus luciferase coding sequence was amplified from 
pLucGR(BS) (24) using the polymerase chain reaction (PCR) (17) and the following primers: forward primer: 5'-TTT TAA GGA TCC ATG ATG AAG AGA GAG AAA AAT GTT G-3' and the reverse primer: 5'-AA TTA ATC TAG AAG TTT AGA ACT CTT CTC CAG-3', where the underlined sequences denote the recognition sites for $B a m \mathrm{HI}$ and $X b a \mathrm{I}$, respectively. The streptavidin-coding fragment was amplified from the vector pUC8-SZ (2) using the following PCR primers: forward primer: $5^{\prime}-\mathrm{T}$ TAT ATT CTA GAC CCC TCC AAG GAC TCG-3' and the reverse primer: $5^{\prime}$-T TTT AAT CTA GAT CTA CTG CTG AAC GGC GTC-3', where the underlined sequences represent the recognition sites $\mathrm{XbaI}$ and BgIII, respectively. The PCRs were carried out using Vent ${ }^{\mathrm{TM}}$ polymerase (New England Biolabs, Beverly, MA, USA), and the fragments were purified on a low-gelling temperature agarose gel (FMC BioProducts, Rockland, ME, USA). The PCR fragment containing the luciferase gene was digested with $B a m \mathrm{HI}$ and $X b a \mathrm{I}$ and ligated to pVL1393 (10), which had been cut with the same enzymes and treated with calf intestinal phosphatase (CIP), yielding pVL1393-LucGR. The PCR fragment containing the streptavidin gene was digested with $\mathrm{XbaI}$ and $B g I I \mathrm{I}$ and ligated to pVL1392, which had been cut with the same enzymes followed by CIP treatment. The resulting

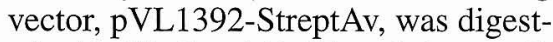
ed with $X b a \mathrm{I}$ and $B g l \mathrm{II}$, and the small, streptavidin-containing fragment was

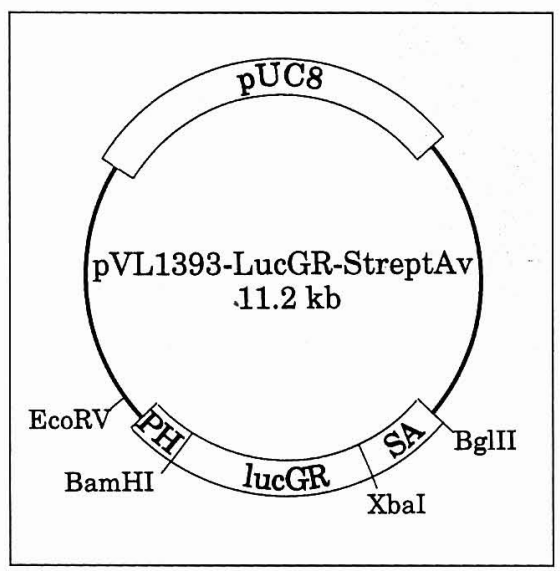

Figure 1. The transfer-plasmid construct pVL1393-LucGR-StreptAv used for generation of the recombinant virus. ligated to vector pVL1393-LucGR, which had been cut with the same enzymes, resulting in transfer vector pVL1393-LucGR-StreptAv (Figure 1). The recombinant DNA techniques and cultivation of the cloning host, $E$. coli MC1061, were performed essentially according to Sambrook et al. (18). Transformation of the bacterial cells was carried out using electroporation (3).

\section{Light Emission Measurements}

At each step of the cloning procedures, bacterial cells were measured for their luminescence by taking $100 \mu \mathrm{L}$ of the $E$. coli MC1061 miniprep culture and adding $50 \mu \mathrm{L}$ of substrate solution (1 mM D-luciferin [BioTools Oy, Turku, Finland], $100 \mathrm{mM}$ Na-citrate, $\mathrm{pH}$ 5.0). Light emission was detected with a BioOrbit Oy 1250 manual luminometer (Turku, Finland). The lightemitting bacterial clones $(5-10 \mathrm{mV}$ at $\mathrm{OD}_{600}$ of 1) were collected and used for restriction analyses.

Similarly, light emission measurements were used for generating the recombinant baculoviruses as recently described (14). The Sf9 cells infected with wild-type baculovirus (strain E2), or the recombinant viruses VL1392LucGR (6), VL1392-ProtA-LucGR (15) or VL1393-LucGR-StreptAv, were grown as monolayer cultures in 24-well plates (Nunc, Roskilde, Denmark) at a density of $3 \times 10^{5}$ cells per well. The infected cells were harvested at 12,24 , 36,48 and $72 \mathrm{~h}$ post infection (p.i.). Aliquots (1-10 $\mu \mathrm{L})$ of the cell suspensions (measurements in vivo) or cell lysates (measurements in vitro) were mixed with $200 \mu \mathrm{L}$ substrate solution (in vivo: $1 \mathrm{mM}$ D-luciferin in $100 \mathrm{mM}$ Na-citrate buffer, $\mathrm{pH} 5.0$; in vitro: luciferase monitoring reagent [BioTools Oy] containing D-luciferin and ATP) and measured as described previously $(6,15)$. Purified firefly luciferase (Sigma Chemical) was used as a standard in all experiments.

\section{Sample Preparation}

The samples exposed for analyses in vitro were prepared essentially as described previously (15). Briefly, at indicated time points p.i., cells were washed once with phosphate-buffered saline (PBS) (pH 7.4) and cytoplasmic extracts prepared using ice-cold disruption buffer (50 mM Tris- $\mathrm{HCl}$ [pH 7.6], 1 mM EDTA, $1 \%$ Nonidet $^{\circledR}$ P40, $15 \%$ glycerol). Lysates were kept on ice for $15 \mathrm{~min}$, sonicated 3 times for $5 \mathrm{~s}$ (MSE 100; Measurement Scientific Equipment, London, England, UK) and clarified by centrifugation at $12000 \times g$ for 10 min. Light emission measurements were performed both from the soluble as well as the insoluble fractions. In the latter case, the precipitates were carefully overlaid with the same buffer once to eliminate the residual soluble supernatant fraction, after which they were resuspended several times prior to the light emission measurements.

\section{Binding to Biotinylated Acrylic Beads}

Acrylic beads labeled with biotin (Sigma Chemical) (binding capacity 1 $\mathrm{mg}$ biotin $/ 25 \mathrm{mg}$ beads) were incubated in buffer A (50 mM Tris- $\mathrm{HCl} \mathrm{pH} \mathrm{7.4,}$ $1 \mathrm{mM}$ EDTA, 15\% glycerol) and washed 3 times with the same buffer (1 $\mathrm{mL} / 25 \mathrm{mg}$ beads) prior to the experiments. For each sample, $12.5 \mathrm{mg}$ of beads in $200 \mu \mathrm{L}$ buffer A were mixed with lysates corresponding to 60000 cells. Samples were agitated for $1 \mathrm{~h}$ at room temperature (RT). Beads were collected by centrifugation at $280 \times g$ for $2 \mathrm{~min}$, washed 3 times with $1 \mathrm{~mL}$ of buffer $\mathrm{A}$ and resuspended in $400 \mu \mathrm{L}$ buffer A for light emission measurements. Supernatants were centrifuged at $280 \times \mathrm{g}$ for an additional $2 \mathrm{~min}$ to ensure the absence of beads. Amounts of 20-200 $\mu \mathrm{L}$ of sample and $100-500 \mu \mathrm{L}$ of substrate were used for the light emission measurements performed as described above.

\section{Sodium Dodecyl Sulfate Polyacryl- amide Gel Electrophoresis (SDS- PAGE)}

Cytoplasmic extracts of cells infected with the recombinant viruses and the wild-type baculovirus were prepared as described above and mixed with an equal volume of $2 \times$ Laemmli sample buffer (7). Samples $(20 \mu \mathrm{L})$ were boiled for $5 \mathrm{~min}$ and loaded on 10\% SDS polyacrylamide slab gels along with molecular weight markers (BioRad, Hercules, CA, USA). Proteins 
were separated by electrophoresis and visualized by Coomassie Brilliant Blue staining.

\section{Immunoblotting}

Immunoblot procedures were performed essentially as described by Towbin et al. (22). Proteins $(9.3 \mu \mathrm{g}$ of protein A purified human Ig per lane) were separated on $10 \%$ polyacrylamide gels (SDS-PAGE) according to Laemmli (7) and electrophoretically transferred to nitrocellulose membranes in transfer buffer (25 mM Tris, $192 \mathrm{mM}$ glycine, 20\% methanol) using a BioRad Trans-Blot ${ }^{\circledR}$ System. The nitrocellulose sheets were then soaked in blocking buffer (PBS + Tween 20 $[0.05 \%]+2 \%$ skimmed milk [Valio Oy, Helsinki, Finland]) to prevent nonspecific antibody binding (1 h, RT). After two washes (PBS + Tween 20 $[0.05 \%])$, filters were immersed into a biotinylated mouse anti-human Ig antibody solution (Sigma Chemical) (1:500 dilution in PBS + Tween 20 [0.05\%]). After a $36-\mathrm{h}$ incubation at $4^{\circ} \mathrm{C}$ and two washes (PBS + Tween 20 [0.05\%]), indicated dilutions of the recombinant streptavidin-LucGR lysates were added and incubated for $1.5 \mathrm{~h}$ at RT. The filters were washed twice in PBS containing $5 \%$ glycerol followed by the addition of substrate (luciferase monitoring reagent; $1 \mathrm{~mL}$ per filter per lane. Finally, the filters were exposed on Hyperfilm ${ }^{\mathrm{TM}}$-MP (Amersham International, Little Chalfont, Bucks, England, UK) for $1 \mathrm{~h}$.

\section{RESULTS}

\section{Expression of the LucGR-Strepta- vidin Fusion Construct}

Sf9 cells infected with VL1393LucGR-StreptAv, AcMNPV, VL1392LucGR and Ac701-ProtA-LucGR were collected at $48 \mathrm{~h}$ p.i. The protein contents were analyzed by SDS-PAGE and Coomassie blue staining (Figure 2A). A major protein of about $75 \mathrm{kDa}$, corresponding to that of the fusion construct, was clearly identified in cells infected with VL1393-LucGR-StreptAv. A 32$\mathrm{kDa}$ polyhedrin expressed by wildtype, baculovirus-infected cells and a $61-\mathrm{kDa}$ LucGR are seen as somewhat weaker bands in this figure. The chimeric protein, ProtA-LucGR of 80-85 kDa, was not clearly identified in the Coomassie blue-stained gel (15). As can be seen from Figure 2B, the overall expression of the LucGR-
StreptAv fusion protein as a function of time correlated with that of polyhedrin and luciferase (6). Some minor proteins also increasing in intensity are related to viral infection and thus are also seen

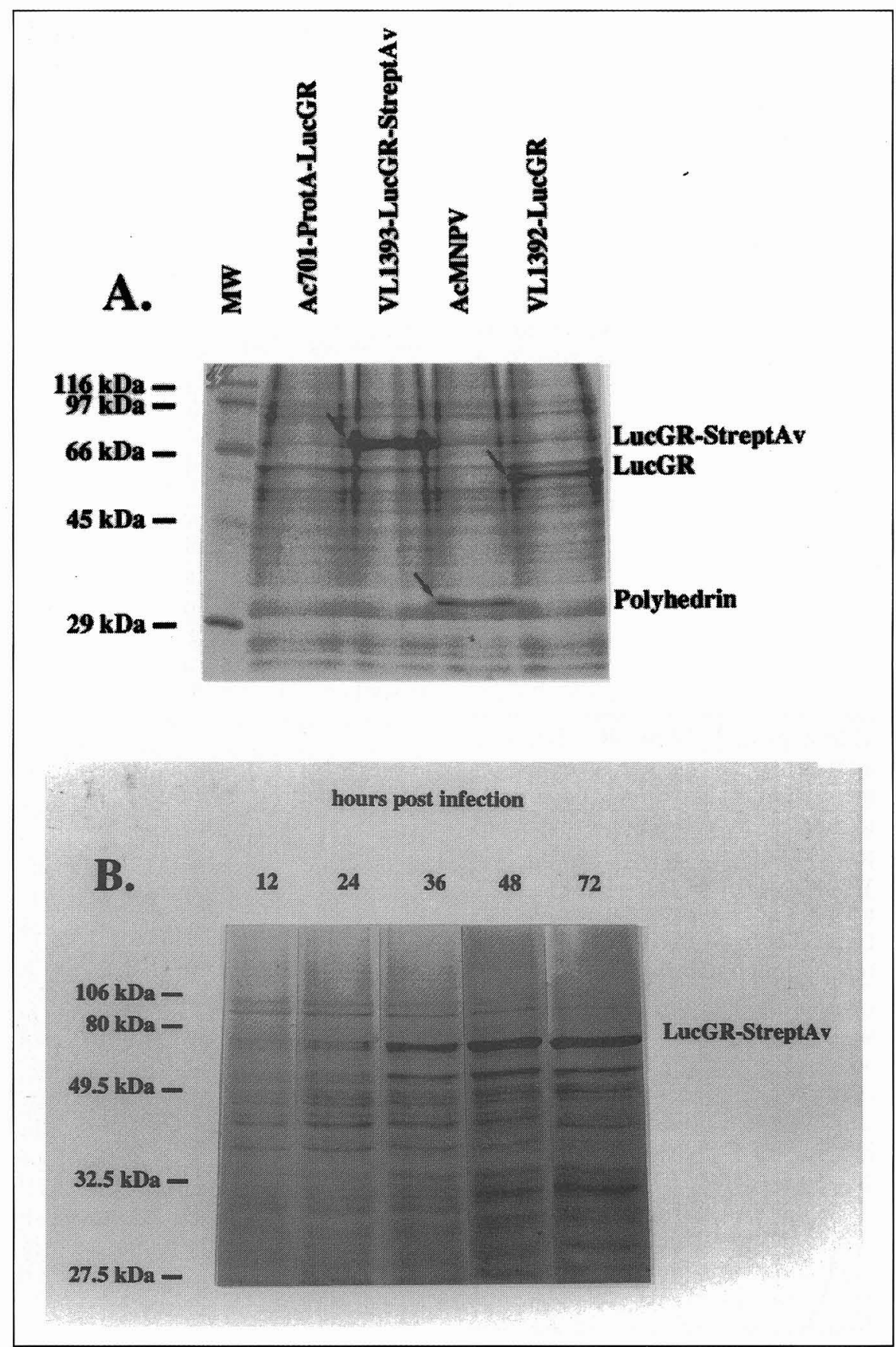

Figure 2. Synthesis of the chimeric protein, LucGR-StreptAv, in Sf9 cells during viral infection. (A) Total cell extracts were prepared at $48 \mathrm{~h}$. p.i. and separated on $10 \%$ vertical SDS-PAGE slab gels followed by Coomassie blue staining. Cells infected with VL1392-LucGR and Ac701-ProtA-LucGR were treated similarly and used as controls. The molecular weight markers in thousands are shown on the left. (B) SDS-PAGE analysis and Coomassie blue staining of Sf 9 cells infected with the recombinant virus, VL1393-LucGR-StreptAv. Cells were collected at 12, 24, 36, 48 and $72 \mathrm{~h}$ p.i. and treated similarly as in Figure 2A. The molecular weight markers in thousands are shown on the left. For details, see the Materials and Methods section. 
in the wild-type, virus-infected cells. None of the indicated proteins were seen in the mock-infected Sf9 cells (data not shown).

\section{Light Emission Measurements}

Cells infected with the recombinant virus, VL1393-LucGR-StreptAv, were collected at 24, 48 and $72 \mathrm{~h}$ p.i. and exposed to in vivo light emission measurements after addition of the substrate, D-luciferin. As shown in Figure $3 \mathrm{~A}$, the amount of light increased with time up to $48 \mathrm{~h}$ p.i. The increase in light production as a function of time correlated with that obtained from cells infected with VL1392-LucGR and Ac701-ProtA-LucGR, respectively. The amounts of light produced by cells infected with VL1393-LucGR-StreptAv $(5-10 \mathrm{mV}$ per cell) were similar when compared to that from cells infected with the unfused VL1392-LucGR construct, whereas the levels of light emitted from cells infected with the Ac701-ProtA-LucGR fusion construct were lower $(0.5-2 \mathrm{mV}$ per cell). The amount of protein produced by Sf 9 cells infected with the respective recombinant viruses was determined by in vitro light emission measurements, where purified authentic firefly luciferase (LucFF) was used as a standard. In Figure 3B, the amount of protein produced by cells infected with the corresponding viruses is shown in $\mu \mathrm{g}$ of protein per $1 \times 10^{6}$ cells. At 72 h p.i., cell cultures infected with the recombinant virus, VL1393-LucGR-StreptAv, produced $127.5 \mu \mathrm{g} / 1 \times 10^{6}$ cells of the chimeric protein. The corresponding expression levels for VL1392-LucGRand Ac701-ProtA-LucGR-infected cells were $239 \mu \mathrm{g} / 1 \times 10^{6}$ cells and $23.5 \mu \mathrm{g} / 1 \times 10^{6}$ cells, respectively.

Binding of the fusion protein, VL1393-LucGR-StreptAv, to immobilized biotin was also analyzed by incubating biotinylated acrylic beads with cytoplasmic extracts prepared from Sf9 cells infected with VL1393-LucGRStreptAv. Expression of the fusion product was performed in insect cell culture medium lacking biotin. Light emission measurements revealed that about $50 \%$ of the fusion protein was precipitated with the biotinylated beads (data not shown). The biotinylated acrylic beads did not precipitate any light-emitting protein from cytoplasmic extracts prepared from cells infected with VL1392-LucGR or Ac701-ProtALucGR, which were used as controls.

\section{Detection of Biotinylated Mouse Anti-Human Ig Antibodies}

The specific reactivity of the baculovirus-expressed chimeric protein

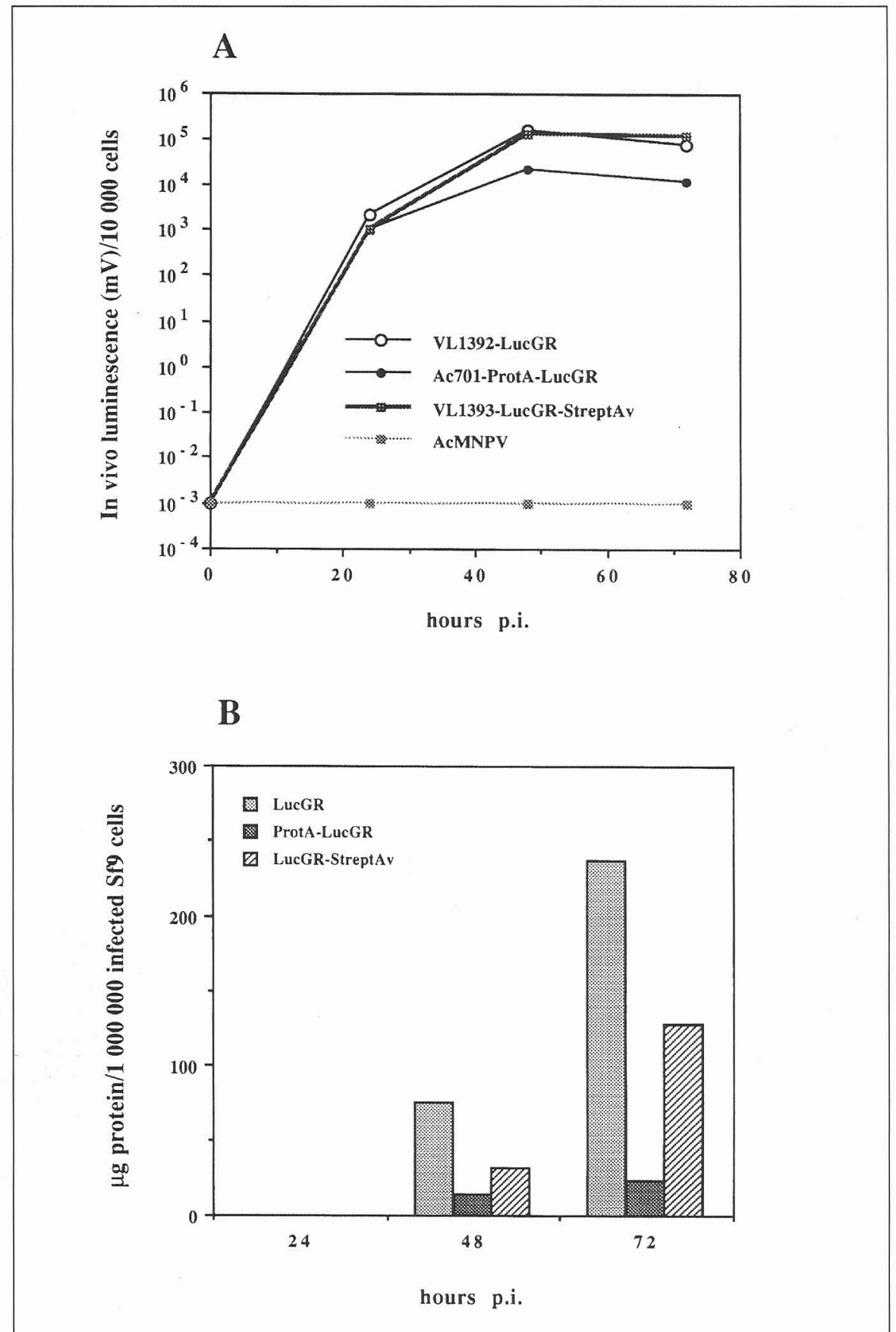

Figure 3. Light emission measurements of Sf9 cells infected with the following recombinant viruses: VL1393-LucGR-StreptAv, VL1392-LucGR, Ac701-ProtA-LucGR and wild-type AcNPV. (A) Cells were collected at 24, 48 and $72 \mathrm{~h}$ p.i., kept intact and used for light emission studies after addition of the substrate D-luciferin (in vivo). Cells infected with wild-type baculovirus (AcMNPV) served as a control. (B) Cells were collected at 24, 48 and $72 \mathrm{~h}$ p.i., disrupted and exposed to light emission measurements after addition of the substrate (in vitro). The amount of recombinant protein was determined using authentic purified firefly luciferase (LucFF; $100 \mathrm{ng} / \mathrm{mL}$ ) as a standard (for details, see Materials and Methods). 
product was further evaluated by immunoblotting. Purified human Ig molecules were separated on SDS-PAGE slab gels and transferred to nitrocellulose membranes as described above. The corresponding proteins were allowed to react with biotinylated mouse anti-human Ig antibodies. These antibody conjugates were then exposed to cytoplasmic extracts containing LucGR-StreptAv and LucGR, respectively. As shown in Figure 4, a protein band with an apparent molecular weight of about $50 \mathrm{kDa}$, corresponding to the heavy-chain portion of the complete Ig molecule, was clearly visible when the proteins were incubated with the extract containing LucGR-StreptAv. This protein band was not detectable with an extract containing LucGR alone.

\section{DISCUSSION}

The strong binding capacity of streptavidin to D-biotin makes it an attractive molecule for many biotechnological and biomedical applications. The gene encoding streptavidin has been expressed in $E$. coli. In a study performed by Sano and Cantor (19), a truncated version of the corresponding gene was constructed and fused to protein A. In another study by Thompson and Weber (21) where a synthetic streptavidin gene was expressed, the yield of active protein after cell disruption, inclusion body solubilization and refolding appeared to be $3 \mathrm{mg} / \mathrm{L}$. In both studies, however, the main difficulty was to obtain soluble and biologically active protein. Streptavidin has, in addition, been expressed as well as secreted from Bacillus subtilis cells (13). In that study, the yield of recombinant streptavidin in minimal biotin-free medium was $20 \mathrm{mg} / \mathrm{L}$.

Here, we report the expression of a biologically functional genetic fusion containing coleopteran insect luciferase and bacterial streptavidin. The chimeric protein was produced in lepidopteran insect cells by using the baculovirus technology, where the first amino acid (D) at the mature amino terminus of streptavidin was linked to the last amino acid (L) of the carboxyl terminus of insect luciferase. The two coding sequences were linked together using an engineered $\mathrm{Xba \textrm {I }}$ recognition site yielding only one additional amino acid (L) between the domains. According to the light emission measurements, the Sf9 cell line was able to produce 127.5 $\mu \mathrm{g}$ of functionally active luciferasestreptavidin fusion protein per one million of infected Sf9 cells. These determinations were carried out using purified authentic luciferase from the firefly, P. pyralis, as a standard. This is in agreement with the data obtained from the SDS-PAGE analyses where the production levels were compared to polyhedrin expressed by wild-type AcMNPV (20). In addition, the lightemitting properties of the chimeric protein were almost identical compared with recombinant luciferase (LucGR). As shown by in vitro experiments (indicating the total amount of protein produced in milligrams), the Protein ALucGR fusion protein was produced at significantly lower levels as compared with LucGR or LucGR-StreptAv (Figure $3 \mathrm{~B}$ ). This may be partly explained by the fact that a different baculovirus transfer vector was used to generate the corresponding recombinant virus (11).
The chimeric protein product described here appeared to bind specifically to vitamin D-biotin. This was shown by precipitating the fusion protein with biotinylated acrylic beads. Close to $50 \%$ of the protein was precipitated with these beads according to the light emission measurements. Furthermore, biotinylated anti-human immunoglobulins bound to human immunoglobulins on nitrocellulose sheets could be detected using the light-emitting properties of this protein and $\mathrm{X}$-ray film. Interesting$1 y$, about half of the recombinant protein was soluble at $36 \mathrm{~h}$ p.i. when the infected cells were grown in ordinary insect cell culture medium. Expression of the fusion protein in culture medium lacking biotin appeared to improve the solubility (data not shown).

Due to the sensitive monitoring procedures by which luciferase activity can be detected, these enzymes represent interesting alternatives to the currently used genetic reporters as well as marker enzymes, e.g., for diagnostic use. Since streptavidin has been frequently used in a number of applica-

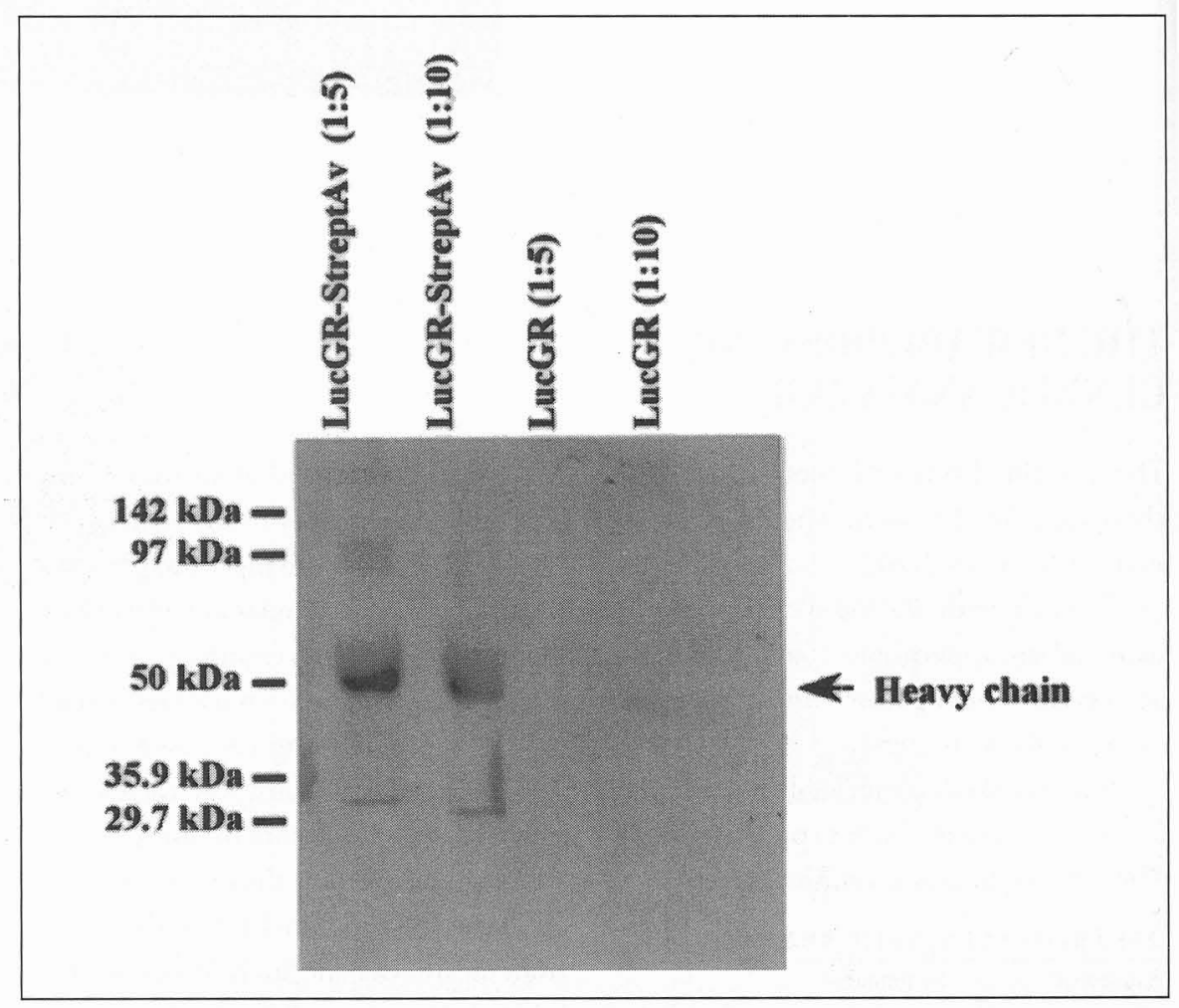

Figure 4. Immunoblot analysis of human immunoglobulins using biotinylated mouse anti-human Ig antibodies and the luciferase-streptavidin fusion protein (LucGR-StreptAv). An amount of 9.3 $\mu \mathrm{g}$ of protein A purified human Ig was separated per lane. Dilutions of the recombinant fusion protein, LucGR-StreptAv, are indicated in the figure (for details, see Materials and Methods). 
tions such as isolation of biotinylated macromolecules and labeling of biologically active materials, it was considered an attractive fusion partner (23). Together the data presented here show that recombinant baculoviruses can be used to produce a functional fusion protein containing the entire coding sequences of luciferase and streptavidin in lepidopteran insect cells. The dual biological activity of the corresponding protein product may be of value within several fields of the biosciences as well as diagnostic medicine.

\section{ACKNOWLEDGMENTS}

We are grateful to Dr. Max Summers for providing the baculovirus transfer vectors, Dr. Takeshi Sano for the streptavidin gene and Nina Tuominen for technical assistance. We also want to thank Dr. Edward Bayer, Dr. Markku Kulomaa and Dr. Meir Wilchek for interesting discussions and valuable comments. This study was funded by the Technology Development Centre of Finland (TEKES), the Academy of Finland, the Ella and Georg Ehrnrooth, the Sigrid Juselius and the Magnus Ehrnrooth foundations.

\section{REFERENC ES}

1.Airenne, K., P. Sarkkinen, E.L. Punnonen and M.K. Kulomaa. 1994. Production of recombinant avidin in Escherichia coli. Gene 144:75-80.

2.Argarana, C.E., I.D. Kuntz, S. Birken, R. Axel and C.R. Cantor. 1986. Molecular cloning and nucleotide sequence of the streptavidin gene. Nucleic Acids Res. 14:18711892.

3.Dower, W.J., J.F. Miller and C.W. Ragsdale. 1988. High efficiency transformation of $\mathrm{E}$. coli by voltage electroporation. Nucleic Acids Res. 16:6127-6145.

4.Hasnain, S.E. and B. Nakhai. 1990. Expression of the gene encoding firefly luciferase in insect cells using a baculovirus vector. Gene 91:135-138.

5.Jha, P.K., B. Nakhai, P. Sridhar, G.P. Talwar and S.E. Hasnain. 1990. Firefly luciferase, synthesized to very high levels in caterpillars infected with a recombinant baculovirus, can also be used as an efficient reporter enzyme in vivo. FEBS 274:24-26.

6.Karp, M., K. Åkerman, C. Lindqvist, A. Kuusisto, P. Saviranta and C. Oker-Blom. 1992. A sensitive model system for in vivo monitoring of baculovirus gene expression in single infected insect cells. Bio/Technology 10:565-569.

7.Laemmli, U.K. 1970. Cleavage of structural proteins during the assembly of the head of bacteriophage T4. Nature 227:680-685.

8.Lampinen, J., L. Koivisto, M. Wahlsten, P. Mäntsälä and M. Karp. 1992. Expression of luciferase genes from different origins in $\mathrm{Ba}$ cillus subtilis. Mol. Gen. Genet. 232:498-504.

9.Lindqvist, C., M. Karp, K. Åkerman and C. Oker-Blom. 1994. Flow cytometric analysis of bioluminescence emitted by recombinant baculovirus-infected insect cells. Cytometry 15:207-212.

10.Luckow, V.A. 1991. Cloning and expression of heterologous genes in insect cells with baculovirus vectors, p. 97-153. In A. Procop, R.K. Bajpai and C.S. Ho (Eds.), Recombinant DNA Technology and Applications. McGrawHill, New York.

11.Luckow, V.A. and M.D. Summers. 1988. Trends in the development of baculovirus expression vectors. Bio/Technology 6:47-55.

12.Miller, L.K. 1988. Baculoviruses as gene expression vectors. Annu. Rev. Microbiol. 42:177-179.

13.Nagarajan, V., R. Ramaley, H. Albertson and M. Chen. 1993. Secretion of streptavidin from Bacillus subtilis. Appl. Environ. Microbiol. 59:3894-3898.

14.Oker-Blom, C., C. Jansson, M. Karp, C. Lindqvist, J.-M. Savola, J. Vlak and K. ̊̊kerman. 1993. Functional analysis of the human a2c-C4 adrenergic receptor in insect cells expressed by a luciferase-based expression vector. Biochim. Biophys. Acta 1076:269275 .

15.Oker-Blom, C., A.-M. Suomalainen, K. Åkerman, Z. Qi, C. Lindqvist, A. Kuusisto and M. Karp. 1993. A baculovirus-expressed fusion protein containing the antibody-binding domain of protein $\mathrm{A}$ and insect luciferase. BioTechniques 14:800-809.

16.O'Reilly, D.R., L.K. Miller and V.A. Luckow. 1992. Baculovirus Expression Vectors - A Laboratory Manual. W.H. Freeman, New York.

17.Saiki, R.K., S. Scharf, F. Faloona, K.B. Mullis, G.T. Horn, H.A. Erlich and N. Arnheim. 1985. Enzymatic amplification of $\beta$ globin genomic sequences and restriction site analysis for diagnosis of sickle cell anemia. Science 230:1350-1354.

18.Sambrook, J., E.F. Fritch and T. Maniatis. 1989. Molecular Cloning: A Laboratory Manual, 2nd ed. Cold Spring Harbor Laboratory Press, Cold Spring Harbor, NY.

19.Sano, T. and C.R. Cantor. 1991. Expression vectors for streptavidin-containing chimeric proteins. Biochem. Biophys. Res. Commun. 176:571-577.

20.Summers, M.D. and G.E. Smith. 1987. A manual of methods for baculovirus vectors and insect cell culture procedures. Tex. Agric. Exp. Sta. Bull. No. 1555. Texas A\&M University.

21.Thompson, L.D. and P.G. Weber. 1993. Construction and expression of a synthetic streptavidin-encoding gene in Escherichia coli. Gene 136:243-246.

22.Towbin, H., T. Staehelin and J. Gordon. 1979. Electrophoretic transfer of proteins from polyacrylamide gels to nitrocellulose sheets: procedure and some applications. Proc. Natl. Acad. Sci. USA 76:4350-4354.

23.Wilchek, M. and E.A. Bayer. 1989. Applications to avidin-biotin technology: literature survey. Methods Enzymol. 184:14-45.

24.Wood, K.V., A.Y. Lam, H.H. Seliger and W.D. McElroy. 1989. Complementary DNA coding click beetle luciferases can elicit bioluminescence of different colors. Science 244:700-702.

Received 1 May 1995; accepted 21 September 1995.

\section{Address correspondence to:}

Christian Oker-Blom

VTT Biotechnology and Food Research

Otaniemi

FIN-02150

Espoo, Finland 